Cite this: Nanoscale, 2013, 5, 581

\title{
Polymer reinforcement using liquid-exfoliated boron nitride nanosheets
}

\author{
Umar Khan, ${ }^{a}$ Peter May, ${ }^{a}$ Arlene O'Neill, ${ }^{a}$ Alan P. Bell, ${ }^{\text {ab }}$ Elodie Boussac, \\ Arnaud Martin, ${ }^{a}$ James Semple ${ }^{a}$ and Jonathan N. Coleman ${ }^{* a}$
}

We have exfoliated hexagonal boron nitride by ultrasonication in solutions of polyvinylalcohol in water. The resultant nanosheets are sterically stabilised by adsorbed polymer chains. Centrifugation-based sizeselection was used to give dispersions of nanosheets with aspect ratio (length/thickness) of $\sim 1400$. Such dispersions can be used to produce polyvinylalcohol-BN composite films. Helium ion microscopy of fracture surfaces shows the nanosheets to be well dispersed and the composites to fail by pull-out. We find both modulus, $Y$, and strength, $\sigma_{\mathrm{B}}$, of these composites to increase linearly with volume fraction, $V_{\mathrm{f}}$, up to $V_{\mathrm{f}} \sim 0.1 \mathrm{vol} \% \mathrm{BN}$ before falling off. The rates of increase are extremely high; $\mathrm{d} Y / \mathrm{d} V_{\mathrm{f}}=670 \mathrm{GPa}$ and $\mathrm{d} \sigma_{\mathrm{B}} / \mathrm{d} V_{\mathrm{f}}=47 \mathrm{GPa}$. The former value matches theory based on continuum mechanics while the latter value is consistent with remarkably high polymer-filler interfacial strength. However, because the mechanical properties increase over such a narrow volume fraction range, the maximum values of both modulus and strength are only $\sim 40 \%$ higher than the pure polymer. This phenomenon has also been observed for graphene-filled composites and represents a serious hurdle to the production of high performance polymer-nanosheet composites.

Received 5th October 2012

Accepted 15th November 2012

DOI: $10.1039 / c 2 n r 33049 k$

www.rsc.org/nanoscale assumption is justified because, for solution processed thin films such as those studied here, planar fillers are known to adopt in-plane alignment. ${ }^{2}$ The parameter $\eta_{\mathrm{LY}}$ is the so-called length efficiency factor which reflects the dependence of reinforcement on platelet length and increases from 0 and 1 with increasing platelet aspect ratio (length/thickness, $L / t$, see below). This expression suggests that the degree of reinforcement can be characterised by the rate of increase of modulus with volume fraction i.e. $\mathrm{d} Y / \mathrm{d} V_{\mathrm{f}}$. This parameter has been used by a number of authors as a figure of merit to assess reinforcement found when adding nano-fillers to polymers. ${ }^{3,4}$ It is clear from eqn (1) that $\mathrm{d} Y / \mathrm{d} V_{\mathrm{f}}$ can never be larger than $Y_{\mathrm{F}}$, demonstrating an upper limit to reinforcement which is set by the properties of the platelet.

Since the 1950's, a succession of materials have been studied. Discontinuous composites of polymers filled with planar materials such as glass, aluminium diboride and silicon carbide flakes were among the first to be studied and generally demonstrated significant improvements in modulus. ${ }^{1}$ For example, probably the most common planar reinforcement of those days, glass platelets, showed linear increases in modulus up to $V_{\mathrm{f}} \sim 70 \mathrm{vol} \%$, resulted in $\mathrm{d} Y / \mathrm{d} V_{\mathrm{f}} \approx$ $70 \mathrm{GPa}$, very close to the modulus of the glass itself. ${ }^{1}$ We note that some of early results were very impressive. Boron carbide platelets gave linear increases in modulus up to $V_{\mathrm{f}} \sim 50 \mathrm{vol} \%$, with values of $\mathrm{d} Y / \mathrm{d} V_{\mathrm{f}} \approx 430 \mathrm{GPa},{ }^{1}$ again reasonably close to the modulus of boron carbide. ${ }^{5,6}$ However, while this value is particularly high, the boron carbide platelets were made by an 
expensive and complex route, ruling this out as a scalable technology.

More recently much work has been done to study the reinforcing properties of clay platelets. ${ }^{7,8}$ This is largely because these fillers are extremely cheap. However, with the exception of a small number of reports, ${ }^{9}$ reinforcement values have not been exceptional. For example, to our knowledge, the highest published rate of increase of modulus with clay mass fraction was for composites of montmorillonite in nylon-6, yielding a relatively high rate of increase of modulus with mass fraction, $M_{\mathrm{f}}$, of $\mathrm{d} Y / \mathrm{d} M_{\mathrm{f}}=42 \mathrm{GPa}^{10}{ }^{10}$ Here the modulus increased linearly with mass fraction to at least $M_{\mathrm{f}}=7 \mathrm{wt} \%$. While these results are reasonably impressive, the vast majority of other reports on clays show much lower reinforcement. ${ }^{9}$ There are probably two reasons for this. As mentioned above, $\mathrm{d} Y / \mathrm{d} V_{\mathrm{f}}$ can never be larger than the modulus of the filler., ${ }^{\mathbf{1 1 1}}$ Clay platelets typically have modulus $\sim 200 \mathrm{GPa}$ (ref. 9, 12 and 13) making this a crude upper limit for $\mathrm{d} Y / \mathrm{d} V_{\mathrm{f}}$. Secondly, exfoliated clay platelets used in composite reinforcement are generally small in lateral dimension, predominately $<1 \mu \mathrm{m} .{ }^{9}$ This can result in low values of $\eta_{\mathrm{LY}},{ }^{2}$ further depressing the reinforcement. As such, very large rates of increase of modulus for clay filled composites are not expected. Moreover, clay platelets are not expected to be particularly strong. While it is hard to find information on the platelet strength, one report gives the tensile strength of mica to be $250 \mathrm{MPa},{ }^{14}$ considerably lower even than glass platelets $(\sim 1 \mathrm{GPa}) .{ }^{1}$ Thus, we do not expect to create ultra-strong composites using clay as a filler.

However, in recent years a new planar reinforcement has become available which combines the superior mechanical properties of boron carbide with low cost, approaching that of clays. That material is of course exfoliated graphite-graphene (graphite powder costs $\sim \$ 5$ per $\mathrm{kg}$, similar to the price of organically modified nanoclays suitable for composite formation). A number of reports have described reinforcing polymers with graphene. Very good reinforcement values have been achieved with $\mathrm{d} Y / \mathrm{d} V_{\mathrm{f}}$ approaching $1000 \mathrm{GPa} .^{2,3,15,16}$ This is close to the reported modulus of individual graphene monolayers themselves. ${ }^{\mathbf{1 7}}$ However, it is worth noting that such levels of reinforcement can only be achieved using exfoliated flakes with large lateral size and aspect ratio considerably above $1000 .^{2}$

Graphite is just one of a large number of layered crystals, some of which may have similarly impressive mechanical properties. In particular, boron nitride (BN) nanosheets (BNNSs) are expected to have mechanical properties similar to graphene, ${ }^{18}$ and are potentially interesting fillers for reinforcing plastics. ${ }^{19,20}$ Furthermore, it has recently been shown that a wide range of layered crystals, including $\mathrm{BN}, \mathrm{MoS}_{2}, \mathrm{WS}_{2}$ etc., can be exfoliated cheaply and easily by sonication in certain solvents to give high aspect ratio nanosheets. ${ }^{\text {20-24}}$ More recently, such nanosheets have been produced by sonicating layered crystals in polymer solutions. The exfoliated nanosheets are then sterically stabilised by adsorbed polymer chains. ${ }^{25}$ Such polymer-nanosheet dispersions are useful as a starting point for the production of solution processed composites, ${ }^{2,26}$ allowing us to investigate whether exfoliated nanosheet materials could act as effective nanoscale reinforcements. We address this question in this paper by preparing composites of exfoliated boron nitride nanosheets in polyvinyl alcohol matrices. We employ centrifugation-based size selection to obtain predominately large area nanosheets with mean aspect ratio of $\sim 1400$. Such flakes allow the formation of composites with values of $\mathrm{d} Y / \mathrm{d} V_{\mathrm{f}}$ of up to 670 $\mathrm{GPa}$, similar to values found for graphene composites. We also observe considerable increases in tensile strength. However, we find important limitations to the practical use of these materials as reinforcing agents.

\section{Experimental procedure}

Polyvinylalcohol (PVA, J. T. Baker, $M_{\mathrm{w}}=77000-79000 \mathrm{~g} \mathrm{~mol}{ }^{-1}$ ) was dissolved in deionised water at a concentration of $20 \mathrm{mg}$ $\mathrm{ml}^{-1}$ by reflux at $100{ }^{\circ} \mathrm{C}$. Hexagonal Boron Nitride (Saint Gobain h-BN, high purity, Average crystallite size $<50$ micron) powder was added to $60 \mathrm{ml}$ of this solution at a concentration of $20 \mathrm{mg}$ $\mathrm{ml}^{-1}$. This mixture was sonicated overnight using a sonic tip (GEX600 at 25\% of $600 \mathrm{~W}$ for 12 hours using $5 \mathrm{~s}$ on $5 \mathrm{~s}$ off pulsation with flat head probe). After sonication the sample was centrifuged at 1000 RPM for 45 minutes (Hettich Mikro 22R) and the supernatant was removed from the sediment. The exfoliated BN flakes produced by this procedure were used as a control sample (no size selection). Under these circumstances, the supernatant contains water, PVA and small exfoliated flakes (lateral size typically $<1 \mu \mathrm{m}$ ) while the sediment contains a mixture of PVA, exfoliated flakes with larger lateral size and unexfoliated h-BN crystallites. ${ }^{2,23,27}$ It is well known that larger flakes result in more efficient reinforcement. ${ }^{\mathbf{1} 2}$ Thus, to extract the larger flakes from the sediment, ${ }^{2,23,27}$ it was topped up using fresh PVA- $\mathrm{H}_{2} \mathrm{O}$ solution and then mildly sonicated (Branson 1510E-MT sonic bath) for 3.5 hours. This solution was then centrifuged at a lower rate of 500 RPM for 22 minutes to remove any unexfoliated h-BN crystallites, and the supernatant collected. Under such circumstances, the BN flakes dispersed in the supernatant are mostly quite large with a minor population of small flakes. In order to minimise the fraction of small flakes, the supernatant was then centrifuged at a higher rate of 3000 RPM for 3 hours, separating the small flakes in the supernatant from the larger flakes in the sediment. The sediment collected from this process was then dried for 3 hours in an oven at $60^{\circ} \mathrm{C}$, and this dried powder was used as the filler material for composite formation.

The dried powder described above contains relatively large BN flakes and some residual PVA. Thermogravimetric analysis (see ESI $\dagger$ ) was carried out on this powder (Perkin Elmer Pyris TGA, $35-1000{ }^{\circ} \mathrm{C}$ range at $10{ }^{\circ} \mathrm{C} \min ^{-1}$ heating rate) to find the ratio of $\mathrm{BN}$ to PVA allowing accurate determination of $\mathrm{BN}$ mass fractions in the final composites. This showed the BN content of the powder to be $84-92 \%$ depending on the run.

This PVA-BN powder was then redissolved in water at a total solids concentration of $1 \mathrm{mg} \mathrm{ml} \mathrm{m}^{-1}$. This dispersion was then blended with a PVA solution of known concentration $(30 \mathrm{mg}$ $\mathrm{ml}^{-1}$ ) to give a range of composite dispersions. The final BN mass fraction (i.e. $\left.M_{\mathrm{f}}=m_{\mathrm{BN}} /\left(m_{\mathrm{BN}}+m_{\mathrm{PVA}}\right)\right)$ was controlled by the relative volumes of PVA-BN and PVA solutions during blending. A range of mass fractions, $M_{\mathrm{f}}$, from $0 \%$ to $1 \%$ were produced. All 
blending was carried out using micropipettes resulting in a high degree of accuracy in the final mass fractions.

In all cases, the composite dispersions were diluted slightly with water to ensure they contained a total solid mass of close to $80 \mathrm{mg}$ (PVA and BN) in a solvent volume of $5 \mathrm{ml}$. After blending and dilution, they were again sonicated in the same bath as before for 4 hours. The dispersion quality was examined by dropping a few drops of solution onto a holey carbon grid (400 mesh) and then viewing using a Jeol 2100 TEM at $200 \mathrm{kV}$. To form films, the dispersions were dropcast into Teflon trays and left overnight in a vacuum oven at $60{ }^{\circ} \mathrm{C}$ with a pressure of 900 mbar. The resultant dried films were $\sim 35$ microns thick and were cut into strips of width $2.25 \mathrm{~mm}$ and tested using a Zwick Roell Tensile tester with a $100 \mathrm{~N}$ load cell at a strain rate of $5 \mathrm{~mm}$ $\min ^{-1}$. For each mass fraction, at least 5 strips were measured and the mechanical properties averaged. Differential scanning calorimetry (DSC, not shown) was carried out on the films using a Perkin Elmer diamond DSC $\left(-5{ }^{\circ} \mathrm{C}\right.$ to $160{ }^{\circ} \mathrm{C}$ range at $20{ }^{\circ} \mathrm{C}$ $\min ^{-1}$ heating rate) to confirm that excess PVA crystallisation $^{28,29}$ had not been nucleated by the presence of the BN nanosheets. This data showed no systematic change in PVA crystallinity on addition of BN. The mass fraction was converted to volume fraction, $V_{\mathrm{f}}$, taking densities of $1300 \mathrm{~kg} \mathrm{~m}^{-3}$ for PVA and $2290 \mathrm{~kg} \mathrm{~m}^{-3}$ for BN powder. Helium ion microscopy was performed on film fracture surfaces with a Zeiss Orion Plus. The working distance was $\sim 5 \mathrm{~mm}$ and a $20 \mu \mathrm{m}$ aperture was used. The beam current was $0.5 \mathrm{pA}$ with a tilt of $15^{\circ}$. We note that the angle between fracture surface and beam was poorly defined as the fracture surface was not flat and not well perpendicular to the film plane.

\section{Results and discussion}

Our first attempts to reinforce polyvinyl alcohol with exfoliated BN used flakes prepared using a very basic exfoliation procedure $^{21}$ (referred to as "control sample" in the Experimental procedure) which gave relatively small flakes (flake length and thickness; $\sim 540 \mathrm{~nm}$ and $\sim 1.2 \mathrm{~nm}$, see ESI $\dagger$ ). The resultant composites showed relatively low levels of reinforcement (see ESI $\dagger$ ). Based on previous results for graphene-reinforced composites, ${ }^{2}$ we realised the mechanical properties were limited by the flake size. To address this problem we applied controlled centrifugation to the control dispersion to select exfoliated BN nanosheets with larger lateral sizes (see Experimental procedure). ${ }^{2,23,27}$

The dispersed, size-selected BN nanosheets were analysed using transmission electron microscopy. Typical images of nanosheets are shown in Fig. 1A-D. Note that the image quality is slightly impaired by the presence of adsorbed PVA. As the nanosheet size has a significant effect on the mechanical properties of composites, ${ }^{2}$ efforts were made to estimate flake dimensions. We measured the length, $L$, and width, $w$, of 132 nanosheets by analysing TEM images. The length data is presented as a histogram in Fig. 1E. The nanosheets lengths range from $\sim 200 \mathrm{~nm}$ to $\sim 7 \mu \mathrm{m}$ with a mean of $\langle L\rangle=1.35 \pm 0.1 \mu \mathrm{m}$. The average nanosheet width was $\langle w\rangle=1.0 \pm 0.1 \mu \mathrm{m}$. In almost all cases, these images show objects that are clearly multilayers.
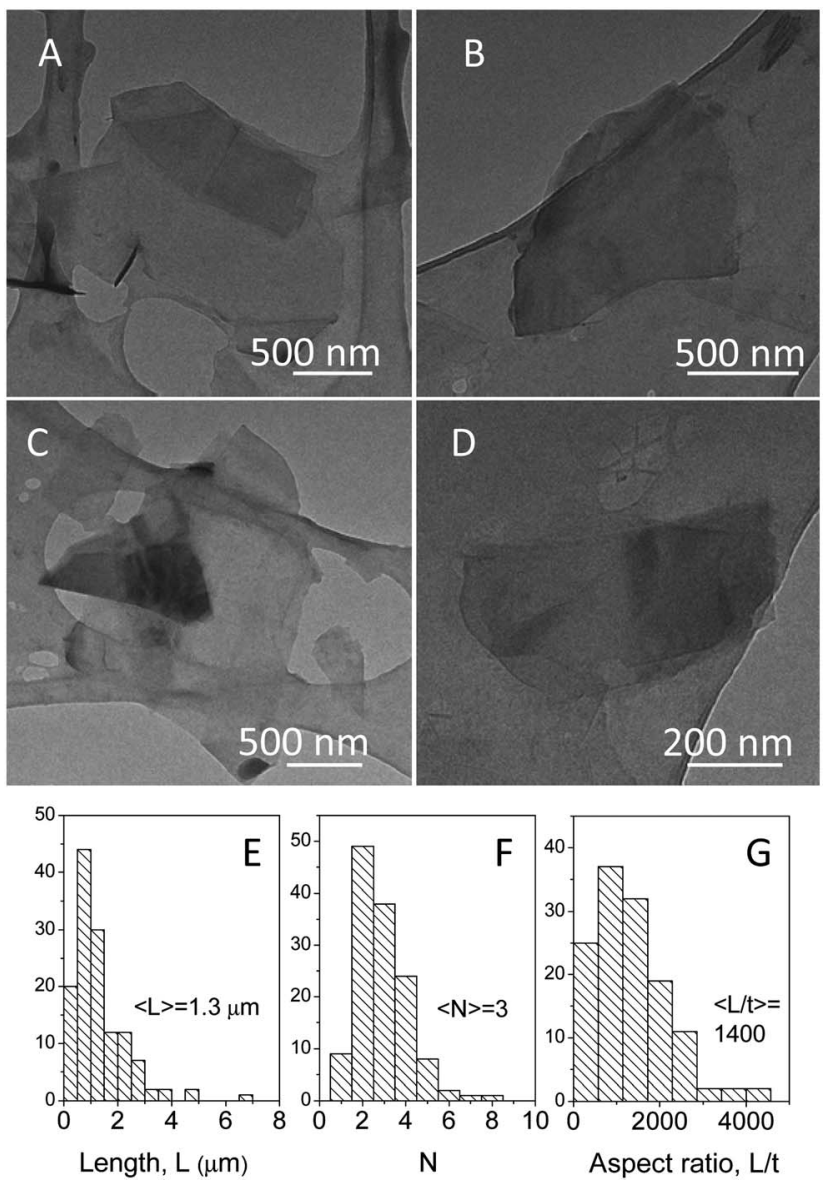

Fig. 1 (A-D) TEM images of liquid exfoliated BN nanosheets. (E-G) Histograms of measured values for flake length, estimated number of layers per flake and flake aspect ratio (length/thickness). 132 flakes were analysed to generate this data.

In most cases, it is possible to estimate the number of layers per flake by counting the edges of the constituent monolayers. ${ }^{30}$ In a small minority of cases, only one edge was visible suggesting the presence some monolayers. The estimated number of layers per flake, $N$, is plotted as a histogram in Fig. 1F. The mean was found to be $\langle N\rangle=3$, very similar to reports for exfoliated graphene..$^{30} \mathrm{NB}$ this implies a mean flake thickness, $t$, of $\langle t\rangle \approx 1 \mathrm{~nm}$. The aspect ratio defined as flake length over thickness, $L / t$, is an important parameter in reinforcement of composites and is shown in Fig. 1G. The mean flake aspect ratio was $\langle L / t\rangle=1400$.

These size-selected BN flakes were used to make composites as described in the methods section. Briefly, dispersions of exfoliated BN and PVA in water with a range of BN mass fractions were poured into Teflon moulds and the water evaporated to give free standing films. In contrast to polymer-graphene composite films, BN-PVA films were largely transparent with a slight milky hue as shown in Fig. 2A. We investigated the dispersion of the BNNS within the composite by examining a fracture surface (broken under tensile stress) with $\mathrm{He}$ ion microscopy. This technique is ideal for this sort of application as it is very effective at imaging insulating surfaces without the need for a metal coating. Shown in Fig. 2B is an image of a 

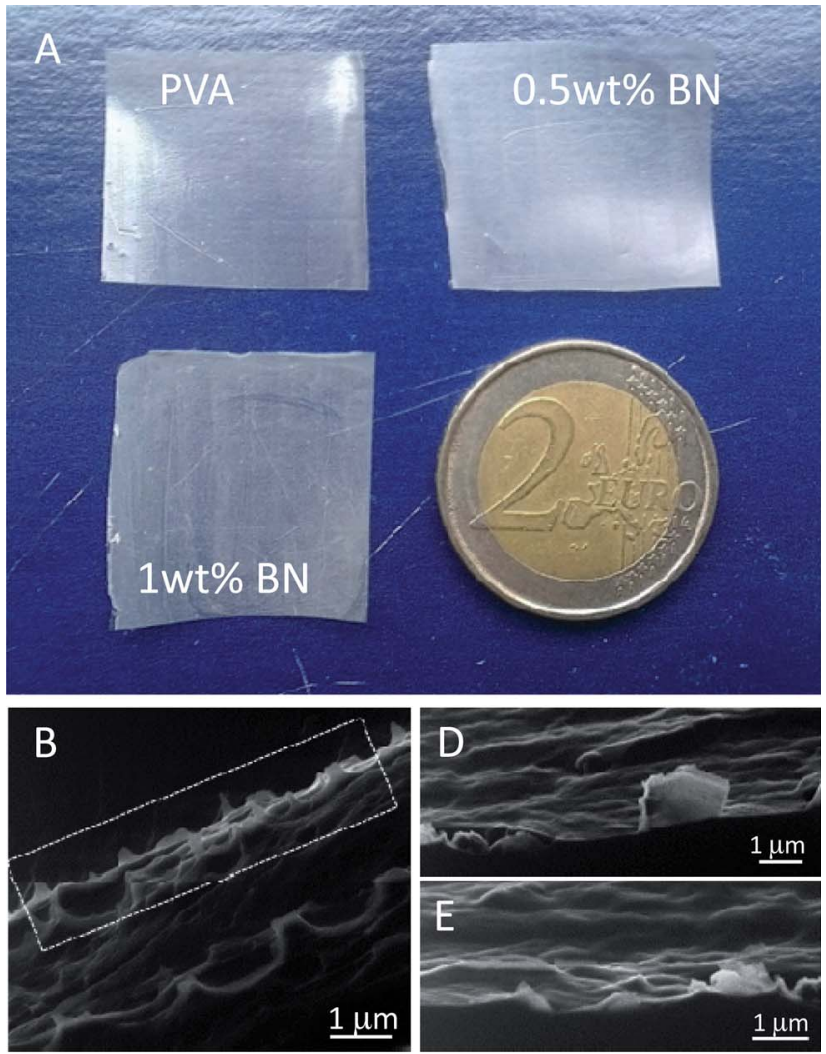

$1 \underline{\mu \mathrm{m}}$
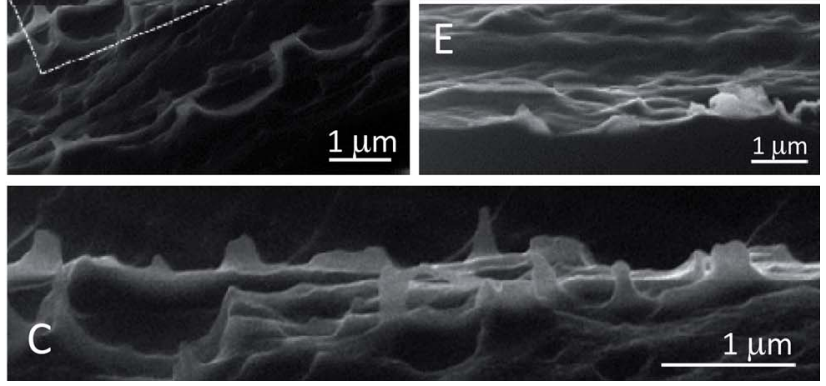

Fig. 2 (A) Photograph of three of the composite films studied in this work. (B) Helium ion microscope image of the fracture surface of a $0.1 \mathrm{wt} \%$ PVA-BNNS composite. Large numbers of well-dispersed, well-exfoliated nanosheets can be seen protruding. (C) Magnified image of the portion of B bounded by the dashed line. ( $D$ and $E$ ) Helium ion microscope image of the fracture surface of a 1 wt\% PVA-BNNS composite. Note the aggregated BNNS.

fracture surface of a $0.1 \mathrm{wt} \%$ composite (0.056 vol\%). Large numbers of BNNS can be seen protruding. These can be seen in more detail in the magnified image in Fig. 2C. For comparison, fracture surfaces of a $1 \mathrm{wt} \%$ composite are shown in Fig. 2D and E. Here aggregated BNNSs can be clearly seen. This indicates that aggregation effects occur at higher BNNS loading levels. In addition, these images suggest the nanosheets to be aligned in the plane of the polymer film.

We measured the tensile mechanical properties of the PVA-BN composites for a number of BN mass fractions as well as a PVA-only reference sample. Representative stress-strain curves for the PVA and the best performing composite are shown in Fig. 3A. It is clear from this data that both modulus and strength of these composites increase on addition of small quantities of exfoliated BN.

We can analyse this data in more detail by plotting the modulus and strength, as extracted from the stress-strain curves, as a function of BN volume fraction. This data is shown in Fig. 3B and C. The modulus increases approximately linearly
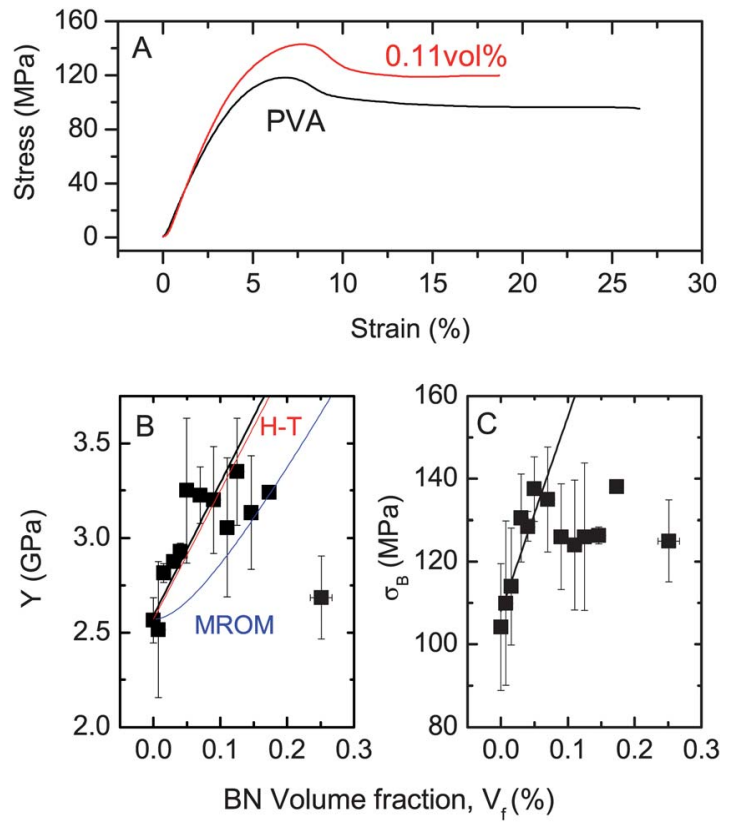

Fig. 3 (A) Representative stress-strain curves for the polymer-only and one of the best performing composite samples. (B) Young's modulus and (C) ultimate tensile strength plotted as a function of BN volume fraction. In both (B) and (C) the solid lines are linear fits to the data for $V_{f}<0.12$ vol\% and 0.06 vol\% respectively. In (B) the blue and red lines represent predictions of the modified rule of mixtures (MROM, eqn (1)-(3)) and the Halpin-Tsai equations ( $\mathrm{H}-\mathrm{T}$, eqn (4) and (5)) respectively.

with filler volume fraction from $\sim 2.5 \mathrm{GPa}$ for the PVA film to $\sim 3.4 \mathrm{GPa}$ for $V_{\mathrm{f}}=0.12 \mathrm{vol} \%$ sample. Such reinforcement is similar to that typically observed for graphene reinforced composites $^{\mathbf{1 5}}$ and relatively large compared to clay reinforced composites. ${ }^{9}$ This is followed by a fall-off in modulus at higher volume fractions. Such behaviour has been observed previously for polymer-nanotube ${ }^{4}$ and polymer-graphene ${ }^{15}$ composites, with the fall-off generally attributed to aggregation of the filler. ${ }^{\mathbf{1 6}}$ This is consistent with the aggregation observed at higher volume fractions and reported in Fig. 2.

The effectiveness of the reinforcing filler can be assessed by the rate of increase of modulus with volume fraction at low loading levels: ${ }^{4} \mathrm{~d} Y / \mathrm{d} V_{\mathrm{f}}$. Applying a linear fit to the low volume fraction portion $\left(V_{\mathrm{f}}<0.12\right.$ vol\%) of the data in Fig. 3B (dashed line) gives a slope of $\mathrm{d} Y / \mathrm{d} V_{\mathrm{f}}=670 \pm 250 \mathrm{GPa}$. This is significantly higher than previous results for $\mathrm{BN}$-polymer composites $\left(\mathrm{d} Y / \mathrm{d} V_{\mathrm{f}} \sim 280 \mathrm{GPa}\right.$ in $\mathrm{PMMA}^{20}$ and $\sim 46 \mathrm{GPa}$ in polybenzimidazole ${ }^{19}$ ) and compares reasonably well with polymers filled with graphene which gives values of $\mathrm{d} Y / \mathrm{d} V_{\mathrm{f}}$ up to $\sim 700$ GPa.,15 Comparisons with other 2-dimensional fillers such as clays, are more appropriately made via $\mathrm{d} Y / \mathrm{d} M_{\mathrm{f}}$ partly because of the significant difference in densities between $\mathrm{BN}$ and most clays but also because the effective volume fraction can be hard to estimate for polymer-clay composites. ${ }^{12}$ The value of $\mathrm{d} Y / \mathrm{d} V_{\mathrm{f}}=670 \pm 250 \mathrm{GPa}$ described above is equivalent to $\mathrm{d} Y / \mathrm{d} M_{\mathrm{f}}=380 \pm 140 \mathrm{GPa}$. To our knowledge, the highest published rate of increase of modulus with clay mass fraction was for composites of montmorillonite in nylon- 6 which gave 
$\mathrm{d} Y / \mathrm{d} M_{\mathrm{f}}=42 \mathrm{GPa}^{10}$ However, the vast majority of reports describing polymer-clay composites show much lower rates of reinforcement. ${ }^{9}$ This clearly shows that with respect to rate of reinforcement with filler volume fraction, BN nanosheets are superior to clay platelets.

We can understand the magnitude of $\mathrm{d} Y / \mathrm{d} V_{\mathrm{f}}$ for these PVA$\mathrm{BN}$ composites by applying theoretical models which describe the dependence of composite modulus on filler volume fraction, $V_{\mathrm{f}}$, and aspect ratio, $L / t$. While a number of such models exist, two of the most common are the modified rule of mixtures (MROM, modified using shear lag theory) ${ }^{1}$ and the Halpin-Tsai equations. ${ }^{31}$ We will apply these models in turn. As described above, the modified rule of predicts the composite modulus to increase linearly with filler volume fraction as described by eqn (1).

However, a detailed analysis based on shear lag theory shows that the length efficiency factor, $\eta_{\mathrm{LY}}$, is given by: ${ }^{1}$

$$
\eta_{\mathrm{LY}}=1-\frac{\tanh (n L / t)}{n L / t}
$$

with

$$
n=\sqrt{\frac{G_{\mathrm{P}} V_{\mathrm{f}}}{Y_{F}\left(1-V_{\mathrm{f}}\right)}}
$$

where $G_{\mathrm{P}}$ is the polymer shear modulus. Taking $Y_{\mathrm{P}}=2.6 \mathrm{GPa}$, we can estimate the shear modulus to be $\sim 0.9 \mathrm{GPa}$ from $Y_{\mathrm{P}}=$ $2 G_{\mathrm{P}}(1+\nu)$ where $\nu_{\mathrm{P}}$ is the polymer Poisson ratio $(\sim 0.5)$. Assuming that $Y_{\mathrm{F}} \sim 750 \mathrm{GPa}$ (ref. 18) and taking $\langle L / t\rangle=1400$ (Fig. 1G) allows us to use eqn (1)-(3) to plot $Y$ as a function of $V_{\mathrm{f}}$ in Fig. 3B. This line does not match the data particularly well, underestimating the data for all volume fractions in the linear region below 0.12 vol\%. This is in some sense surprising as the MROM has previously been shown to describe the performance of polymer-graphene composites very well. ${ }^{2}$

Alternatively, the in-plane mechanical properties of composites filled with in-plane aligned platelets can be described using the Halpin-Tsai equations. Within this model, the composite modulus is given by ${ }^{\mathbf{1 6}}$

$$
Y=Y_{\mathrm{P}}\left[\frac{1+2 V_{\mathrm{f}} \eta L / t}{1-V_{\mathrm{f}} \eta}\right]
$$

where

$$
\eta=\frac{Y_{\mathrm{F}} / Y_{\mathrm{P}}-1}{Y_{\mathrm{F}} / Y_{\mathrm{P}}+2 L / t}
$$

Taking the values for $Y_{\mathrm{P}}, Y_{\mathrm{F}}$ and $\langle L / t\rangle$ described above allows us to use eqn (4) and (5) to plot $Y$ as a function of $V_{\mathrm{f}}$ in Fig. 3B. This time, we get very good agreement between theory and experimental data, particularly for lower volume fractions. The Halpin-Tsai model also agreed very well with the mechanical data found for the composites prepared with un-size selected flakes (see ESI $\dagger$ ). This agreement is significant as it shows that we are achieving reinforcement values consistent with the theoretical limit for BN nanosheets of the aspect ratio produced here. Interestingly, both models are based on continuum mechanics which appear applicable even to nanoscale systems such as those used here. It is also worth noting that both models assume very good interfaces between filler and matrix. Thus the agreement found here implies that the stress transfer at the PVA-BN interface is reasonably good.

Shown in Fig. 3C is the measured composite strength, $\sigma_{\mathrm{B}}$, plotted as a function of $\mathrm{BN}$ volume fraction. As with the modulus, the strength increases linearly from $105 \mathrm{MPa}$ for the PVA to $\sim 140 \mathrm{MPa}$ for $V_{\mathrm{f}}=0.05 \mathrm{vol} \%$ after which the strength falls off. Again, such an increase is similar to the best results observed for graphene reinforced composites ${ }^{2,15}$ and relatively large compared to clay reinforced composites. ${ }^{9}$ Fitting (solid line) gives the slope of the linear region to be $\mathrm{d} \sigma_{\mathrm{B}} / \mathrm{d} V_{\mathrm{f}}=47 \pm 19$ GPa. This is significantly higher than the best strength reinforcement value reported for polymer-graphene composites of $\mathrm{d} \sigma_{\mathrm{B}} / \mathrm{d} V_{\mathrm{f}}=22 \mathrm{GPa} .^{2}$ Moreover, we note that the majority of graphene filled composites have demonstrated $\mathrm{d} \sigma_{\mathrm{B}} / \mathrm{d} V_{\mathrm{f}}<10 \mathrm{GPa}$ (see ref. 2, $\mathrm{ESI} \dagger$ ), making the $\mathrm{BN}$ data all the more impressive. As before, we compare this reinforcement to that found for polymer clay composites via $\mathrm{d} \sigma_{\mathrm{B}} / \mathrm{d} M_{\mathrm{f}}$. The value of $\mathrm{d} \sigma_{\mathrm{B}} / \mathrm{d} V_{\mathrm{f}}=47 \pm 19$ GPa quoted above is equivalent to $\mathrm{d} \sigma_{\mathrm{B}} / \mathrm{d} M_{\mathrm{f}}=27 \pm 11 \mathrm{GPa}$. As far as we are aware, the highest rates of strength increase for polymer-clay composites are $\mathrm{d} \sigma_{\mathrm{B}} / \mathrm{d} M_{\mathrm{f}} \sim 0.4 \mathrm{GPa}$ for montmorillonite in nylon-6 - significantly below the value found here. ${ }^{\mathbf{1 0 , 3 2}}$ Furthermore, as with modulus, most reports show much lower rates of strength increase. ${ }^{9}$

To understand what controls $\mathrm{d} \sigma_{\mathrm{B}} / \mathrm{d} V_{\mathrm{f}}$ in polymer-BN composites it is necessary to consider the failure mechanisms. In general the composite strength can be written as a modified rule of mixtures: ${ }^{1}$

$$
\sigma_{\mathrm{B}}=\left(\eta_{\mathrm{L} \sigma} \sigma_{\mathrm{F}}-\sigma_{\mathrm{P}}\right) V_{\mathrm{f}}+\sigma_{\mathrm{P}}
$$

where $\sigma_{\mathrm{F}}$ and $\sigma_{\mathrm{P}}$ are the strengths of the filler (the nanosheets) and the polymer respectively and $\eta_{\mathrm{L} \sigma}$ is a length efficiency factor. ${ }^{1}$ In fact, the functional form of the length efficiency factor depends on the composite failure mechanism. There are two possibilities, depending on whether the aspect ratio of the nanosheets is above or below a critical value. ${ }^{33}$ When external stress is applied to a nanosheets-containing composite, some of the applied stress is transferred to the nanosheets. How much depends on the interfacial stress transfer and the aspect ratio of the nanosheets. If the aspect ratio is above a critical value, enough stress can be transferred to the nanosheets to result in their fracture. If the aspect ratio is below the critical value, failure of the composite tends to be via fracture of the nanosheets-polymer interface i.e. pull-out. Following the standard derivation of the critical aspect ratio for rod-like fillers, ${ }^{\mathbf{1 1}}$ we can easily derive the critical aspect ratio for planar fillers to be:

$$
\left(\frac{L}{t}\right)_{\mathrm{c}}=\frac{\sigma_{\mathrm{F}}}{\tau}
$$

where $\tau$ is the average stress transferred from polymer to nanosheets. The strength of BNNSs has not been measured experimentally. However, theoretical studies suggest it to be as high as $85 \mathrm{GPa}^{34}$ This can be compared with the strength of graphene ${ }^{17}$ of $\sim 130 \mathrm{GPa}$ and individual $\mathrm{MoS}_{2}$ monolayers of 23 $\mathrm{GPa}^{2}$ We assume the theoretical value is a reasonable 
approximation for a defect free BNNS. In addition, the stress transfer at the BN-PVA interface is unknown. However, Gong et al. previously estimated the stress transfer at a graphene-PVA interface to be $\sim 1 \mathrm{MPa} .{ }^{35}$ Assuming this value can be used as an approximate estimate of the stress transfer in our case, we can use eqn (7) to make a very crude estimate that $(L / t)_{\mathrm{c}} \sim 85000$. Given that the measured value of $L / t$ is $\sim 1400$, this implies that the $\mathrm{BN}$ flakes used in this work have aspect ratio well below the critical value. A similar conclusion was previously reported for graphene-BN composites. ${ }^{2}$

If the nanosheet aspect ratio is well below the critical value, the composites will fail via pull-out. This means that rather than the nanosheets breaking, the polymer-nanosheet interface fails, resulting in a portion of the nanosheet protruding from the fracture surface. This can be seen to be the case in Fig. 2C. On average, the protruding length should be one quarter of the total nanosheet length. ${ }^{28}$ We can test this by estimating the length of the protruding nanosheets in Fig. 2C. Crudely assuming the fracture surface to be inclined at an angle of $\sim 45^{\circ}$ to the beam, we estimate the mean protruding length to be $300 \pm 150 \mathrm{~nm}$. This is very close to the value of $\langle L\rangle / 4=340 \pm 25$ $\mathrm{nm}$ measured by TEM, confirming that the data is consistent with failure by pull-out.

As such, we can analyse the data using the interfacial failure model. In this case the form of the strength efficiency factor is such that the composite strength is given by: ${ }^{2}$

$$
\sigma_{\mathrm{B}} \approx\left[\frac{\tau_{\mathrm{B}}}{4}\left(\frac{\langle L\rangle+\langle w\rangle}{\langle t\rangle}\right)-\sigma_{\mathrm{P}}\right] V_{\mathrm{f}}+\sigma_{\mathrm{P}}
$$

where $\tau_{\mathrm{B}}$ is the interfacial shear strength. Taking the measured value of $\mathrm{d} \sigma_{\mathrm{B}} / \mathrm{d} V_{\mathrm{f}}$, and the dimensions discussed above gives a value of $\tau_{\mathrm{B}}=80 \pm 32 \mathrm{MPa}$. This is considerably larger than the value estimated for graphene-PVA composites of $\sim 30 \mathrm{MPa} .^{2}$ That this value is so high, indeed higher than the reported value of the PVA shear strength, ${ }^{36}$ is rather surprising and requires further study. However, this value of $\tau_{\mathrm{B}}$, coupled with eqn (8), was also found to describe data for the composites prepared with un-size selected flakes (see ESI $\dagger$ ). This suggests that for the size selected flakes, $\mathrm{d} \sigma_{\mathrm{B}} / \mathrm{d} V_{\mathrm{f}}$ (and so $\mathrm{d} \sigma_{\mathrm{B}} / \mathrm{d} M_{\mathrm{f}}$ ) is larger than that found for other 2-dimensional fillers, most likely because the polymerfiller interface is particularly strong for BN-PVA. However, we note that this interfacial strength may be particular to the nature of the PVA-BN interaction and may not apply for other matrices. Indeed, composites prepared from BNNS in PMMA displayed $\mathrm{d} \sigma_{\mathrm{B}} / \mathrm{d} M_{\mathrm{f}}=1.6 \mathrm{GPa},{ }^{20}$ considerably below the value reported here. In order to successfully use BNNS to reinforce polymers other than PVA, it may be necessary to functionalise the surface of the BNNS to improve the interfacial strength (and indeed stress transfer). While BN has long been thought too inert to functionalise, recent reports have shown that the basal plane of BNNS can be covalently functionalised with polymers. ${ }^{37}$ Thus it may be possible to tailor the surface chemistry of $\mathrm{BN}$ to reinforce a wide range of polymers. Preliminary results suggest such strategies to be reasonably successful. ${ }^{37-39}$ In addition, it is worth noting that even if high $\tau_{\mathrm{B}}$ values can be routinely achieved, because of the form of eqn (8), flakes with relatively large lateral size will be required to achieve large values of $\mathrm{d} \sigma_{\mathrm{B}} / \mathrm{d} V_{\mathrm{f}}$.
However, it should be pointed out that while $\mathrm{d} Y / \mathrm{d} V_{\mathrm{f}}$ and $\mathrm{d} \sigma_{\mathrm{B}} / \mathrm{d} V_{\mathrm{f}}$ are both high, the maximum values of composite modulus and strength are not very high (3.2 GPa and $140 \mathrm{MPa}$ respectively). This is because, as is observed for graphene filled composites, ${ }^{2}$ the mechanical properties of the composites only increase with increasing volume fraction at very low volume fractions before peaking. This is in stark comparison to polymer-clay composites where the mechanical properties may increase (albeit slowly) up to clay contents of $>10 \% .{ }^{9}$ It is not certain what limits the peak mass fraction of polymer-BN (or graphene-polymer) composites, although it is probably aggregation-related. This implies that both the flake aspect ratio and the balance of nanosheets-solvent-polymer interaction energies are important. In any case further work is required to understand this system and to extend the region of increasing modulus and strength to higher volume fractions resulting in much high composite properties.

\section{Conclusions}

In conclusion, we have prepared composites of liquid exfoliated boron nitride nanosheets in a polyvinyl alcohol matrix. We observe considerable reinforcement with maximum increases in modulus and strength of $40 \%$ relative to the pure polymer at volume fraction of only 0.12 vol\%. The rate of increase of modulus with volume fraction is very high and is consistent with models based on continuum mechanics. Similarly, the rate of increase of strength with volume fraction is high, suggesting a very strong BN-PVA interface. However, both modulus and strength increase only over a very limited volume fraction range, with decreasing values observed at higher volume fractions. Thus, while rates of increase are high, the absolute increases in modulus and strength are modest. Similar effects have been observed for graphene reinforced composites. ${ }^{2}$

If unresolved, this will probably be the single biggest obstacle to using high-strength nanosheets for polymer reinforcement. More research is needed to understand the nature of the peak in reinforcement at low volume fraction. In addition, strategies need to be developed to extend the increase in stiffness and strength to much higher volume fractions, leading to stiff, strong composites. For example, maintaining the rates of increase in stiffness and strength measured here up to a volume fraction of just $1 \%$, would result in a composite with modulus of $\sim 7 \mathrm{GPa}$ and strength of $\sim 500 \mathrm{MPa}$. This would represent an extremely useful structural material enabled by very small quantities of nano-material.

The authors would like to acknowledge Science Foundation Ireland, (grant number 07/IN.7/I1772), and ERC grant SEMANTICS for financial support. We also acknowledge the CRANN Advanced Microscopy Lab (AML) for help with Helium ion microscopy.

\section{References}

1 G. E. Padawer and N. Beecher, Polym. Eng. Sci., 1970, 10, 185192. 
2 P. May, U. Khan, A. O'Neill and J. N. Coleman, J. Mater. Chem., 2012, 22, 1278-1282.

3 J. J. Vilatela and D. Eder, ChemSusChem, 2012, 5, 456-478.

4 J. N. Coleman, U. Khan, W. J. Blau and Y. K. Gun'ko, Carbon, 2006, 44, 1624-1652.

5 J. M. Grow and R. A. Levy, J. Mater. Res., 1994, 9, 2072-2078.

6 J. Sun, H. Ling, W. J. Pan, N. Xu, Z. F. Ying, W. D. Shen and J. D. Wu, Tribol. Lett., 2004, 17, 99-104.

7 Y. Fukushima and S. Inagaki, J. Inclusion Phenom., 1987, 5, 473-482.

8 Y. Kojima, A. Usuki, M. Kawasumi, A. Okada, Y. Fukushima, T. Kurauchi and O. Kamigaito, J. Mater. Res., 1993, 8, 11851189.

9 S. Pavlidou and C. D. Papaspyrides, Prog. Polym. Sci., 2008, 33, 1119-1198.

10 T. D. Fornes, P. J. Yoon, H. Keskkula and D. R. Paul, Polymer, 2001, 42, 9929-9940.

11 B. Harris, Engineering Composite Materials, Maney Materials Science, 1999.

12 B. Chen, J. R. G. Evans, H. C. Greenwell, P. Boulet, P. V. Coveney, A. A. Bowden and A. Whiting, Chem. Soc. Rev., 2008, 37, 568-594.

13 L. E. McNeil and M. Grimsditch, J. Phys.: Condens. Matter, 1993, 5, 1681-1690.

14 H. P. Schreiber, Y. B. Tewari and M. R. Wertheimer, J. Appl. Polym. Sci., 1976, 20, 2663-2673.

15 T. Kuilla, S. Bhadra, D. H. Yao, N. H. Kim, S. Bose and J. H. Lee, Prog. Polym. Sci., 2010, 35, 1350-1375.

16 R. J. Young, I. A. Kinloch, L. Gong and K. S. Novoselov, Compos. Sci. Technol., 2012, 72, 1459-1476.

17 C. Lee, X. D. Wei, J. W. Kysar and J. Hone, Science, 2008, 321, 385-388.

18 L. Duclaux, B. Nysten, J. P. Issi and A. W. Moore, Phys. Rev. B: Condens. Matter Mater. Phys., 1992, 46, 3362-3367.

19 Y. Wang, Z. X. Shi and J. Yin, J. Mater. Chem., 2011, 21, 11371-11377.

20 C. Y. Zhi, Y. Bando, C. C. Tang, H. Kuwahara and D. Golberg, Adv. Mater., 2009, 21, 2889-2893.

21 J. N. Coleman, M. Lotya, A. O'Neill, S. D. Bergin, P. J. King, U. Khan, K. Young, A. Gaucher, S. De, R. J. Smith, I. V. Shvets, S. K. Arora, G. Stanton, H. Y. Kim, K. Lee, G. T. Kim, G. S. Duesberg, T. Hallam, J. J. Boland, J. J. Wang, J. F. Donegan, J. C. Grunlan, G. Moriarty, A. Shmeliov, R. J. Nicholls, J. M. Perkins, E. M. Grieveson,
K. Theuwissen, D. W. McComb, P. D. Nellist and V. Nicolosi, Science, 2011, 331, 568-571.

22 G. Cunningham, M. Lotya, C. S. Cucinotta, S. Sanvito, S. D. Bergin, R. Menzel, M. S. P. Shaffer and J. N. Coleman, ACS Nano, 2012, 6, 3468-3480.

23 A. O'Neill, U. Khan and J. N. Coleman, Chem. Mater., 2012, 24, 2414-2421.

24 R. J. Smith, P. J. King, M. Lotya, C. Wirtz, U. Khan, S. De, A. O'Neill, G. S. Duesberg, J. C. Grunlan, G. Moriarty, J. Chen, J. Z. Wang, A. I. Minett, V. Nicolosi and J. N. Coleman, Adv. Mater., 2011, 23, 3944-3948.

25 P. May, U. Khan, J. M. Hughes and J. N. Coleman, J. Phys. Chem. C, 2012, 116, 11393-11400.

26 U. Khan, P. May, A. O'Neill and J. N. Coleman, Carbon, 2010, 48, 4035-4041.

27 U. Khan, A. O'Neill, H. Porwal, P. May, K. Nawaz and J. N. Coleman, Carbon, 2012, 50, 470-475.

28 J. N. Coleman, M. Cadek, R. Blake, V. Nicolosi, K. P. Ryan, C. Belton, A. Fonseca, J. B. Nagy, Y. K. Gun'ko and W. J. Blau, Adv. Funct. Mater., 2004, 14, 791-798.

29 J. N. Coleman, M. Cadek, K. P. Ryan, A. Fonseca, J. B. Nagy, W. J. Blau and M. S. Ferreira, Polymer, 2006, 47, 85568561.

30 U. Khan, A. O'Neill, M. Lotya, S. De and J. N. Coleman, Small, 2010, 6, 864-871.

31 J. C. Halpin and J. L. Kardos, Polym. Eng. Sci., 1976, 16, 344352.

32 Y. Kojima, A. Usuki, M. Kawasumi, A. Okada, T. Kurauchi and O. Kamigaito, J. Appl. Polym. Sci., 1993, 49, 1259-1264.

33 W. D. Callister, Materials Science and Engineering an Introduction, John Wiley, 2007.

34 Q. Peng, W. Ji and S. De, Comput. Mater. Sci., 2012, 56, 11-17.

35 L. Gong, I. A. Kinloch, R. J. Young, I. Riaz, R. Jalil and

K. S. Novoselov, Adv. Mater., 2010, 22, 2694-2697.

36 R. B. Yallee and R. J. Young, Composites, Part A, 1998, 29, 1353-1362.

37 T. Sainsbury, A. Satti, P. May, A. O'Neill, V. Nicolosi, Y. K. Gun'ko and J. N. Coleman, Chem.-Eur. J., 2012, 18, 10808-10812.

38 J. H. Yu, X. Y. Huang, C. Wu, X. F. Wu, G. L. Wang and P. K. Jiang, Polymer, 2012, 53, 471-480.

39 T. Sainsbury, A. Satti, P. May, Z. Wang, I. McGovern, Y. K. Gun'ko and J. Coleman, J. Am. Chem. Soc., 2012, 134, 18758-18771. 